\title{
Network of recurrent events for the Olami-Feder-Christensen model
}

\author{
Tiago P. Peixotd* \\ Instituto de Física, Universidade de São Paulo, Caixa Postal 66318, 05315-970 - São Paulo - Brazil \\ Jörn Davidsen $\dagger^{\dagger}$ \\ Complexity Science Group, Department of Physics \& Astronomy, \\ University of Calgary, Calgary, Alberta T2N 1N4, Canada
}

(Dated: November 20, 2018)

\begin{abstract}
We numerically study the dynamics of a discrete spring-block model introduced by Olami, Feder and Christensen (OFC) to mimic earthquakes and investigate to which extent this simple model is able to reproduce the observed spatiotemporal clustering of seismicty. Following a recently proposed method to characterize such clustering by networks of recurrent events [Geophys. Res. Lett. 33, L1304, 2006], we find that for synthetic catalogs generated by the OFC model these networks have many non-trivial statistical properties. This includes characteristic degree distributions - very similar to what has been observed for real seismicity. There are, however, also significant differences between the OFC model and earthquake catalogs indicating that this simple model is insufficient to account for certain aspects of the spatiotemporal clustering of seismicity.
\end{abstract}

PACS numbers: 89.75.Da,05.65.+b,91.30.Dk,91.30.Px

\section{INTRODUCTION}

Describing and modelling the spatiotemporal organization of seismicity and understanding the underlying physical mechanisms of earthquake triggering have been proven challenging. Inspired by empirical regularities which include selfsimilar scaling laws like the Omori law and the GutenbergRichter law [1, 2], a wealth of mechanisms and models have been proposed including the concepts of a critical earthquake, of self-organized criticality, and more generally of the seismogenic crust as a self-organized complex system requiring a so-called system approach (see, for example, Refs. [3, 4] for a review). Yet, the origin of the observed non-trivial emergent features of earthquake occurrence is still one of the main unresolved problems in the field.

Resolving this issue may require measuring the microscopic state variables - the stress and strain at every point within the earth along active earthquake faults — and their exact dynamics. This is currently impossible. However, the associated earthquake patterns are readily observable making macroscopic approaches based on the concept of spatiotemporal point processes feasible, where the description of each earthquake is reduced to its size or magnitude, its epicenter and its time of occurrence. Describing the patterns of seismicity may shed light on the fundamental physics since these patterns are emergent processes of the underlying many-body nonlinear system.

Recently, such an approach has led to the identification of new properties of the clustering of seismicity in space and time $[5,6,7,8,9,10,11,12,13,14,15,16]$. In particular, the observed spatiotemporal clustering of seismicity suggests that the usual mainshock/aftershock scenario - where each event has at most one correlated predecessor of larger magnitude

*tpeixoto@if.usp.br

tdavidsen@phas.ucalgary.ca
- is too simplistic and that the causal structure of seismicity could extend beyond immediately subsequent events. To quantify such correlations, a general procedure for inferring a plausible causal structure from clusters of localized events has been introduced in Refs. [17, 18]. The approach allows one to study the dynamical organization of spatiotemporal activity in terms of the topology of complex networks [19, 20] and has led to the detection of unexpected statistical features for earthquake catalogs from California. Most importantly, the approach provides a new and independent estimate of the rupture length and its scaling with magnitude.

In this article, we investigate to which extent the simple dynamics of the Olami-Feder Christensen (OFC) model [21] is sufficient to account for the observed spatiotemporal clustering of seismicity as characterized by the above network approach. The OFC model is maybe the simplest model in the class of self-organized critical (SOC) models which exhibit a phenomenology resembling seismicity. This includes the aforementioned Gutenberg-Richter law for the frequencymagnitude distribution [21, 22] and the Omori law for aftershocks [23, 24] as well as some statistical properties of epicenter locations and dynamics [25]. In addition, the OFC model is of special relevance in the context of SOC. Nonequilibrium systems are called self-organized critical if they reach a stationary state characterized by power laws and the absence of characteristic scales - without the need for finetuning an external parameter such as the temperature [26, 27]. This is typically the case for slowly driven systems with fast avalanche-like dissipation events. Unlike the paradigmatic SOC sandpile model [28], where any amount of dissipation is enough to introduce a scale which breaks criticality, the existence of criticality in the OFC model with dissipation is still debated [29, 30, 31, 32, 33, 34].

We show that when the causal structure of the OFC model is studied with the network method mentioned above, it does indeed reveal some similarities to seismicity, such as the degree distributions of the network, and some aspects of the recurrence time and distance statistics. However, there are sev- 
eral important differences — including the absence of a rupture length — that severely hinder its adoption as a plausible (sole) description of the fundamental mechanisms responsible for the spatiotemporal correlations of seismicity. Many of those discrepancies seem to be closely related to the fact that the model is defined on a regular, discrete lattice, and to the existence of quasi-periodic attractors [25]. These features, which are totally absent in seismicity, manifest themselves quite clearly in several aspects of the recurrence network. Also, the recurrence time statistics show a characteristic time due to the fact that the system is uniformly driven.

The outline of our paper is as follows. In Section [I] we review the OFC model, and Section 【 IIImmarizes the network method to search for signs of causal structure in spatiotemporal data. Section IV presents the results obtained for the OFC model, which are compared to real seismicity. We conclude in SectionV

\section{OLAMI-FEDER-CHRISTENSEN MODEL}

The OFC model [21] is inspired by the Burridge-Knopoff spring-block model [35], and is defined on a square lattice of size $L^{2}$. To each site $(i, j)$, a "tension" $z_{i j}$ is assigned, initially chosen at random from the interval $\left[0, z_{c}[\right.$. The entire system is driven slowly, with every $z_{i j}$ increasing uniformly. Whenever a site reaches the threshold tension $z_{i j}=z_{c}$, a relaxation event — called "avalanche" or "earthquake" — starts. The originating site of the avalanche $(i, j)$ is called the epicenter. The dynamics of such an event is as follows: A fraction $\alpha$ of the tension at the epicenter is transferred to each of its four neighbors $z_{i \pm 1, j \pm 1}=z_{i \pm 1, j \pm 1}+\alpha z_{i j}$, and the tension at the site itself is reset $z_{i j}=0$. If the tension at any of the neighboring sites reaches the threshold, $z_{i \pm 1, j \pm 1} \geq z_{c}$, the same toppling rules are applied again. This dynamics continues until there are no more sites in the system with $z_{i j} \geq z_{c}$. Then, the tension increases uniformly again until the next avalanche occurs. Without loss of generality, we set $z_{c}=1$. The total number of topplings during an avalanche is called the "size" of the avalanche $s$. In contrast, the total number of sites that toppled (ignoring multiple topplings of the same site) is called the "area" of the avalanche $a$. The time in the system can be measured either discretely, by the number of events ("iterations"), or continuously, by the cumulative tension injected in the system when it is driven (the so called "natural" time scale). The parameter $\alpha$ defines the level of local conservation of the system. For $\alpha=0.25$ the system is locally conservative and for $\alpha<0.25$ it is dissipative. We consider here only the case of open boundary conditions, i.e., the sites at the border of the lattice transfer tension out of the system (or to an imaginary neighbor), so the system is always globally nonconservative.

After a transient regime, which is increasingly longer for smaller values of $\alpha$, the system reaches a stationary state which has a distribution of event sizes resembling a powerlaw in the tail for the largest, numerically accessible system sizes (see Ref. [34] for an extensive review and the most recent results). For the values of $\alpha \in\{0.18 ; 0.2 ; 0.22\}$ and $L=1000$ we consider here, the power law tail has an unique exponent around -1.8 [30]. Yet, the occurrence of events is not uniformly distributed within the lattice, and tend to happen closer to the boundaries [25, 31]. This "border effect" prevents the event size statistics from obeying finite-size scaling (FSS) with the size of the lattice. Only when events close to the border are ignored and an internal subset of the system is considered, FSS is realized [31]. Interestingly, this border effect does not seem to scale with system size [25]. To accommodate for these observations, we generally restrict our analysis to synthetic catalogs generated by the OFC model which contain only those events involving sites which are all at a distance of at least 100 sites away from the boundary. The effective systems size is thus $L_{\text {bulk }}=800$.

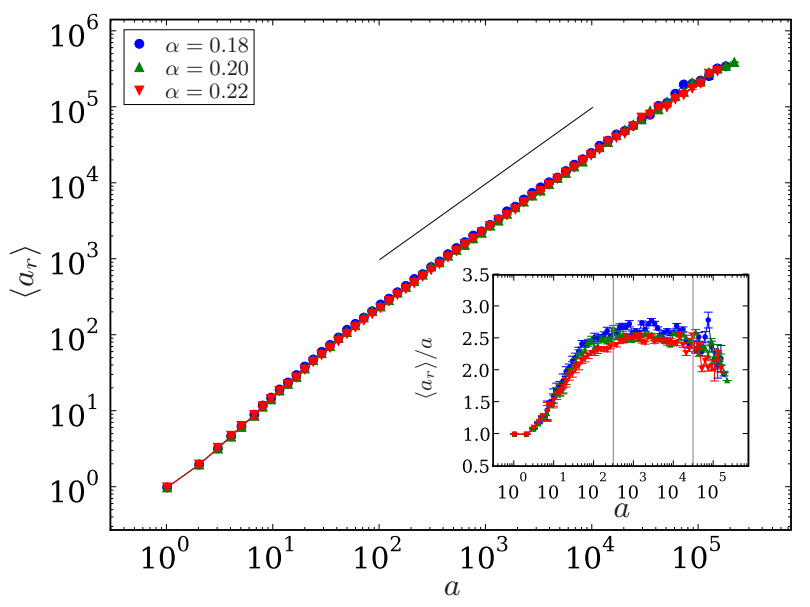

FIG. 1: (Color online) The average area $\left\langle a_{r}\right\rangle$ of the smallest rectangle on the lattice containing a given avalanche of spatial extent $a$ for different values of the dissipation parameter $\alpha$. Only avalanches contained within the bulk, $L_{\text {bulk }}=800$, are considered (see text for details). The solid line corresponds to a linear increase. Inset: Rescaled version indicating that the area scales linearly with $a$ independent of $\alpha$ for $300 \leq a \leq 30000$ (area enclosed by straight lines).

An important aspect in seismicity is the effect of the detection threshold: Earthquake catalogs are only complete above a certain magnitude value which depends on the network of seismometers deployed in a given area. Thus, it is crucial to understand how the statistical properties of seismicity vary with the magnitude threshold. To address this issue in the OFC model, we consider catalogs for different lower threshold values of event sizes $s_{\text {th }}$. Reasonable choices of these values are limited by the particular dynamics of the OFC model and lattice effects. For example, it is well known that events of size one have their own separate dynamics and obey a different statistics [34]. This is easy to justify, since events of size one will occur even if there is no self-organization, i.e., $\alpha=0$. The effects of this particular dynamics and of the discrete lattice can be clearly seen in the spatial shapes of the avalanches as summarized in Fig. 1, The ratio between the average area $\left\langle a_{r}\right\rangle$ of the smallest rectangle which contains an avalanche of area $a$ and $a$ varies significantly with $a$ for all considered values of $\alpha$. Here the average is taken over all events of area $a$. Only for events above a certain size 
TABLE I: Number of events $N$ in the catalogs generated by the OFC model for different values of $\alpha$ and lower size threshold $s_{\mathrm{th}}$.

\begin{tabular}{c|c|c|c}
\hline \hline$s_{\text {th }}$ & $\alpha=0.18$ & $\alpha=0.20$ & $\alpha=0.22$ \\
\hline 300 & 1000000 & 1000000 & 1000000 \\
500 & 641933 & 676324 & 685237 \\
1000 & 327124 & 394235 & 400339 \\
3000 & 107550 & 148311 & 156189 \\
5000 & 60041 & 87102 & 94968 \\
10000 & 22205 & 40208 & 42991 \\
30000 & 2547 & 8432 & 8909 \\
\hline \hline
\end{tabular}

$a_{\text {th }} \sim 10^{2}$, does $a_{r}$ scale linearly with $a$ (see inset of Fig. 1 independent of $\alpha$. Yet, this linear scaling is limited to values of $a<3 \times 10^{4}$ due to the finite system size. Since area and size of an avalanche are basically identical for the parameters of $\alpha$ considered here [40], we only consider catalogs with lower size threshold $s_{\text {th }} \in[300 ; 30000]$ in the following.

To be more specific, each synthetic catalog generated by the OFC model contains $N$ consecutive events or avalanches occurring after the statistically stationary regime has been reached[41] for $L=1000$ and $L_{\text {bulk }}=800$. Each event $k$ is characterized by its size $s_{k}$, its epicenter location $(i, j)_{k}$ and its time of occurrence $t_{k}$ measured in terms of the continuous natural time scale (the discrete avalanche number time scale of the OFC model [36] is basically equivalent). The various catalogs for different values of $\alpha$ and $s_{\text {th }}$ are given in Table $\llbracket$.

\section{NETWORK OF RECURRENCES}

We analyze the catalogs generated by the OFC model according to a recently proposed method which has been proven helpful in characterizing the spatiotemporal clustering of seismicity and in detecting causal signatures between events [17, 18]. The essential idea is to extend the notion of a recurrence to spatiotemporal point processes: An event is defined to be a recurrence of any previous event if it occurred closer in space than all intervening events. Recurrences are therefore record breaking events with respect to distance. By linking each event to its recurrences, a directed network [42] of recurrences is obtained: Each event $a_{k}$, with $k=1, \ldots, N$, is a vertex in the network and a directed edge from $a_{k}$ to $a_{m}$ exists for $k<m$ if $a_{m}$ is a recurrence of $a_{k}$, i.e., if the distance between $(i, j)_{m}$ and $(i, j)_{k}$ is smaller than the distance between $(i, j)_{k}$ and $(i, j)_{k^{\prime}}$ for all events $a_{k^{\prime}}$ with $k<k^{\prime}<m$. This definition assumes that the events are ordered according to their occurrence in time $t_{k}$. Obviously, each recurrence or edge can be characterized by the time interval $t=t_{m}-t_{k}$ between the two connected events $k$ and $m$ and by the spatial distance $l$ between the two. Note that the mapping of the dynamics to a network is entirely data-driven and does not impose any arbitrary space and time scales other than those associated with the given event or earthquake catalog - in contrast, for example, to methods typically used to define aftershocks. Comparing the statistical properties of the network of recurrences for a given catalog to the properties of a network obtained for a random point process without any causal relation between events highlights relevant parts of the underlying causal dynamical process(es) generating the pattern. For the OFC model, such a random process or null model can be obtained by shuffling the entire catalog, containing all events: Shuffle the sizes and the epicenter locations separately while keeping the times of occurrence. Later the $s_{\mathrm{th}}$ threshold can be raised, and the corresponding shuffled versions of each catalog can be obtained. For a random spatiotemporal point process in continuous space and time (CST), several statistical properties of the network of recurrences are even known analytically [18].

\section{RESULTS}

In the following, we do not only compare the network properties of the OFC catalogs with those of their random or shuffled counterparts but also with the network properties found for earthquake catalogs from southern California [17, 18]. The particular focus is on the network topology as summarized, for example, in Refs. [19, 20] and on distributions associated with the temporal and spatial distances between an event and its recurrences.

\section{A. Topological properties}

\section{Network growth}

An important aspect of the analysis of networks and their dynamics is the change in their topology with time. In the case of networks of recurrences, this corresponds to the situation when a given catalog is extended to cover a longer time period and, thus, contains more events. In general, some properties of the network of recurrences depend on its size $N$. For random CST events, one such property is the average degree $\langle k\rangle$ or equivalently the average number of recurrences per event. It grows with the size of the network as $\langle k\rangle \approx \ln (N)$ for $N \gg 1$ [18]. While this is roughly what we find for the shuffled OFC catalogs, the original OFC catalogs show quite a different growth as can be seen in Fig. 2 Independently of the value of $\alpha$, the average degree "saturates" quickly around a value close to 5 , and grows very little even after the catalog is a couple of orders of magnitude larger. When the catalog is shuffled, a portion of the growth can be well represented by $\ln (N)$ but only up to $\sim 10^{4}$ events, after which it grows more slowly.

These observations illustrate one of the main features of the OFC model, namely the repetitive occurrence of events originating at the same location. Obviously, there is a finite probability that the epicenters of two events are identical since the OFC model is defined on a discrete and finite lattice. If this happens, the cascade of recurrences of the first event ends or is "closed" and the number of recurrences of this event does not increase further (see Fig. 3) . If this were exclusively due to the finiteness of the discrete lattice, one would expect this 


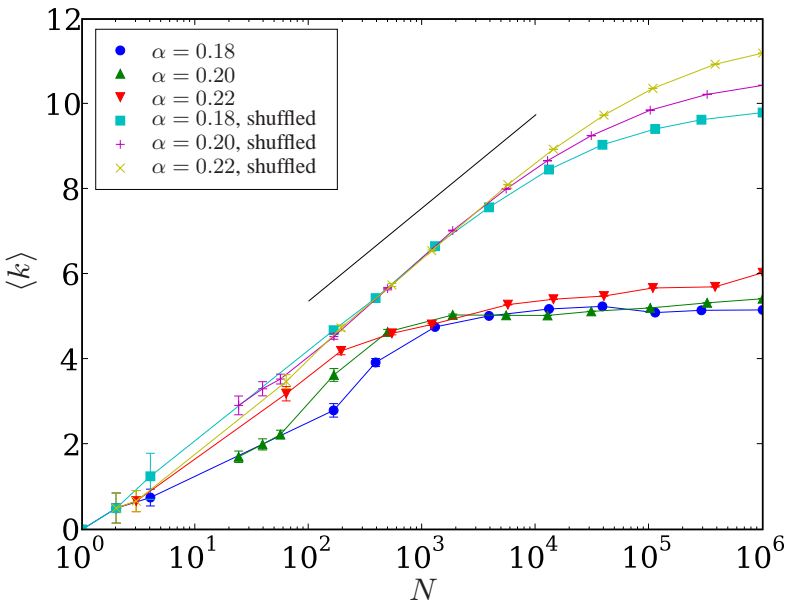

FIG. 2: (Color online) Mean degree $\langle k\rangle$ vs. catalog size $N$ for $s_{\text {th }}=$ 300 and different values of alpha, for the regular and shuffled OFC catalogs. $N=10^{6}$ corresponds to the full catalogs - see Table $\square$ The solid line is of the form $0.95 \ln (N)+$ const.

to happen according to a geometric distribution with average $L_{\text {bulk }}^{2}$. In particular, this effect would not be relevant in the thermodynamic limit $L_{\text {bulk }} \rightarrow \infty$. However, our simulations show that the repeated occurrence of the same epicenter happens much more frequently in the OFC model. In particular, the OFC catalogs for $s_{\text {th }}=300$ in Table \ have $79 \%, 87 \%$ and $92 \%$ closed cascades for $\alpha=0.22,0.20,0.18$, respectively [43]. This is due to marginal synchronization of neighboring sites [37] and the existence of quasi-periodic patterns in the dynamics of the OFC model [25] causing the repeated triggering of epicenters and similar events. Consequently, the average out-degree of networks generated by the OFC model grows rather slowly. Moreover, the growth in $\langle k\rangle$ basically saturates after $\sim 10^{3}$ events as Fig. 2 shows. This is at least two orders of magnitude smaller than the rough estimate of $L_{\text {bulk }}^{2}=6.4 \times 10^{5}$ based on the lattice size.
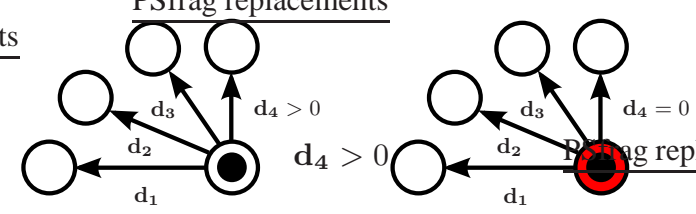

FIG. 3: (Color online) Schematic representation of two cascades of recurrences of size 4 . In both cases $d_{4}<d_{3}<d_{2}<d_{1}$. The cascade on the left is "open", i.e. it is possible for a fifth recurrence to occur, since $d_{4}>0$. This is not the case for the cascade on the right. Since $d_{4}=0$, this cascade is "closed" and no distance will be able to break the record.

When the catalog is shuffled it does not entirely remove the effect. This is because the average frequency of a given epicenter does not change, and the distribution of these frequencies is quite broad, resembling a power law [25]. Yet, the shuffling destroys the relatively short periods of quasi-periodic dynamics and increases in most cases the time it takes for the activity to return to the same epicenter and to close a given cascade of recurrences. Thus, $\langle k\rangle$ is larger and its growth is very close to the predicted behavior for random CST events until the effects of the broad distribution of the epicenters frequencies become important after $\sim 10^{4}$ events.

The network growth described above differs significantly from what is obtained for seismicity, since real epicenters happen on a continuous space and therefore never occur exactly in the same place (save, of course, for limitations in precisely locating the epicenters). Hence, the network growth for seismicity — at least for the given catalog sizes — does not exhibit the "saturation" of the average degree obtained for the OFC model, and grows instead continuously as $\langle k\rangle \approx$ $0.84 \ln (N)$ [18].

\section{Degree distributions}

A more detailed aspect of the network topology is the distribution of in- and out-degrees, $P\left(k_{\text {in }}\right)$ and $P\left(k_{\text {out }}\right)$. In the context of recurrence networks, the out-degree of a vertex corresponds to its number of recurrences while the in-degree is the number of events of which it is a recurrence. As shown in Fig. 4 the out-degree distribution deviates significantly from the random CST case, where a Poisson distribution is expected both for the in- and out-degree distributions [18]. The out-degree distribution seems instead to decay exponentially, with inclination slightly dependent on $\alpha$. The in-degree distribution is closer to a Poissonian. The overall shape of the distributions also does not depend on the choice of the lower size threshold for $300 \leq s_{\text {th }} \leq 30000$ (not shown). When the catalogs are shuffled, the obtained out-degree distribution is very close to a Poissonian (Fig. 5) as expected for the random CST case.

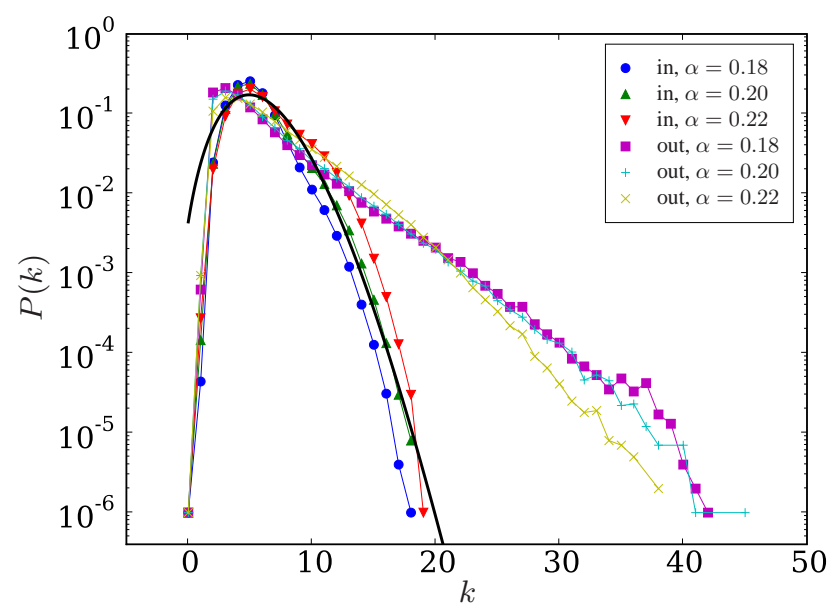

FIG. 4: (Color online) In-degree and out-degree distributions for networks generated by the OFC model for $s_{\text {th }}=300$ and different $\alpha$ 's (see Table【for details). The solid (black) line is a Poisson distribution with the same $\langle k\rangle$ as for $\alpha=0.20$.

The in- and out-degree distributions obtained for the origi- 


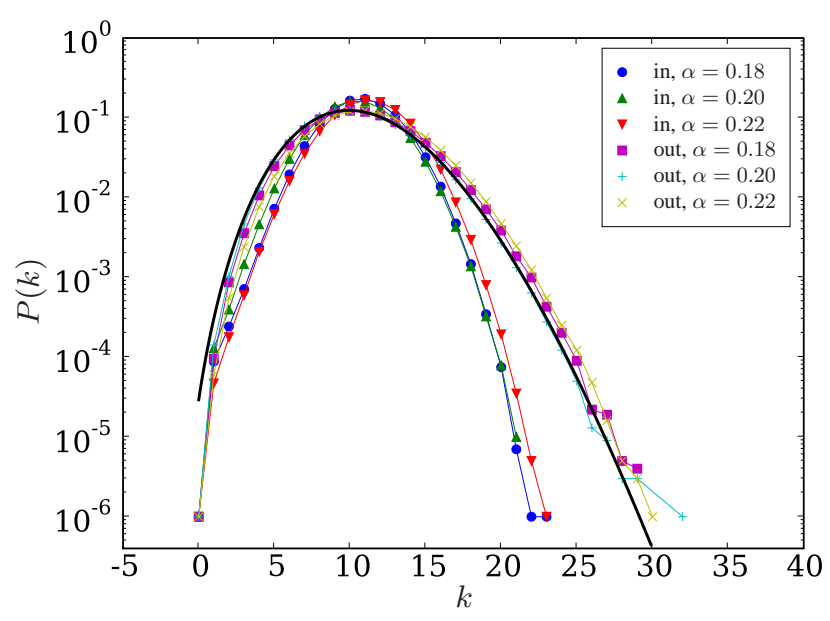

FIG. 5: (Color online) In-degree and out-degree distributions for networks generated from shuffled OFC catalogs with $s_{\text {th }}=300$ and different $\alpha$ 's. The solid (black) line is a Poisson distribution with the same $\langle k\rangle$ as for $\alpha=0.20$.

nal OFC catalogs are basically identical to those for seismicity [18]. The only significant differences are the values of $P(1)$ for the out-degree. For seismicity, it is much more likely to have an event with out-degree one or equivalently an event with a single recurrence. This is related to the finite extension of an earthquake, as characterized by its "rupture length", and the corresponding microscopic dynamics. Thus, our findings suggest the absence of such a length in the OFC model. This is indeed confirmed by other, more direct means discussed in Sec. IVC Nevertheless, the close resemblance of the degree distributions for the OFC catalogs and earthquake catalogs suggests that the OFC model is able to reproduce all other non-trivial gross features of the spatiotemporal clustering of seismicity which are captured by the degree distributions.

\section{Degree-degree correlations}

The network topology can be further characterized by the degree-degree correlations [20]. These correlations indicate how likely vertices of a given in- or out-degree are connected to vertices with another given $k_{\text {in }}$ or $k_{\text {out }}$. We also consider the correlations between $k_{\text {in }}$ and $k_{\text {out }}$ of the same vertex, the single vertex degree-degree correlations. To capture both types of correlations, we measure the average $k_{\text {in }}$ or $k_{\text {out }}$ (either of the neighbors or of the same vertex) where the average is taken over all vertices with a given $k_{\text {in }}$ and $k_{\text {out }}$. For the OFC catalogs, Figs. 6 and 7 show the absence of pronounced nontrivial (single vertex) degree-degree correlations. There are only small qualitative differences between the shuffled and unshuffled catalogs [44] indicating that the degree-degree correlations are not particularly sensitive to the given form of spatiotemporal clustering. This is in agreement with findings for seismicity [18]. Interestingly, the single vertex degree-degree correlation function $\left\langle k_{\text {out }}\right\rangle\left(k_{\text {in }}\right)$ increases with $k_{\text {in }}$ (Fig. 7) - both for the shuffled and the original OFC catalogs. This is different from shuffled earthquake catalogs and especially from random CST processes which show a slight decrease related to the age of a vertex [18]. The deviating behavior of the (shuffled) OFC catalogs is due to the closing of cascades of recurrences mentioned above which largely reduces the correlation between the age of a vertex and its in- and out-degree. Instead, vertices with large $k_{\text {in }}$ correspond to sites with low activity which do not belong to the quasi-periodic attractors. These sites do not experience the aforementioned closing of the cascade of recurrences, and thus have a larger out-degree. The shuffling of the catalog does not alter this since it does not affect the closing of cascades - only the time it takes to close. This reasoning is confirmed by the right panel of Fig.7 which shows the single vertex degree correlation, when only open cascades of recurrences are considered, and the increase is less evident - and disappears completely for the shuffled case.
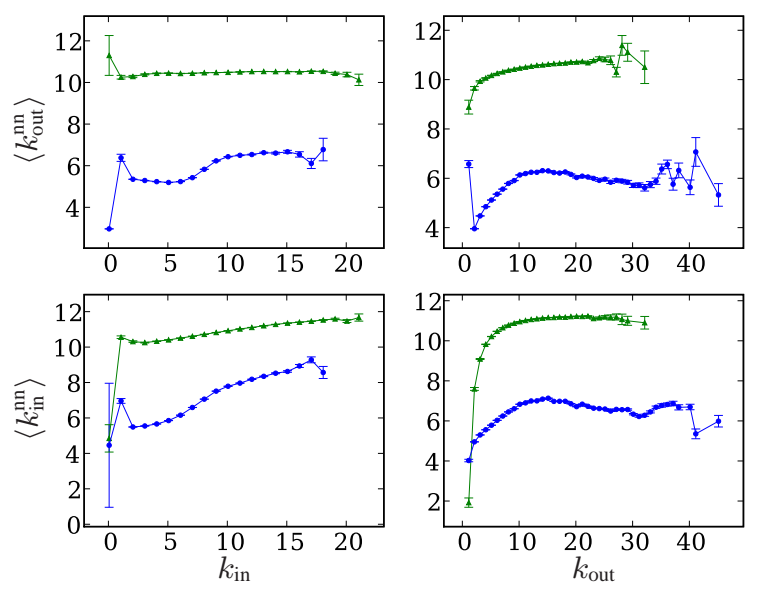

FIG. 6: (Color online) Degree-degree correlations for $\alpha=0.2$ and $s_{\text {th }}=300$. The curves on the bottom (green) are for the original catalog, and on top (blue) for the shuffled catalog. Note that the offset between the curves for the original OFC catalog and the shuffled version is simply due to different mean degrees.
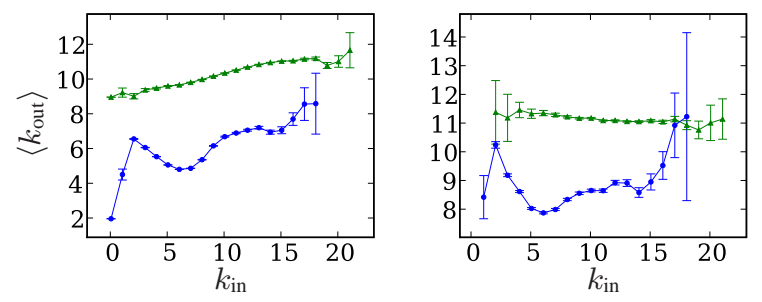

FIG. 7: (Color online) Single vertex degree-degree correlations for $\alpha=0.2$ and $s_{\mathrm{th}}=300$. The left panel shows the results for the original catalogs, and the right panel shows the results for the open cascade of recurrences only. The bottom curves (green) are for the unmodified catalogs, and the top ones (blue) are for the shuffled catalog. 
TABLE II: Clustering coefficients (Eq.(1)) of the recurrence networks generated by the OFC model for different values of $\alpha$ and lower size threshold $s_{\text {th }}=300$. The values of $C$ excluding events with closed cascade of recurrences and for the shuffled OFC catalogs are shown for comparison.

\begin{tabular}{cr|r|r}
\hline \hline$\alpha$ & $C$ & $C$ (open) & $C$ (shuffled) \\
\hline 0.18 & $0.4153(8)$ & $0.2064(8)$ & $0.15411(8)$ \\
0.20 & $0.3910(3)$ & $0.1970(6)$ & $0.15891(8)$ \\
0.22 & $0.3470(3)$ & $0.1891(4)$ & $0.14650(7)$ \\
\hline \hline
\end{tabular}

\section{Clustering coefficient}

The clustering coefficient [20] is another quantity that characterizes the network topology. It quantifies how likely neighbors of the same vertex are connected with each other. In the present context, this refers to the probability that recurrences of the same event are also recurrences of each other.

For all vertices $i$ with out-degree larger than one, the local clustering coefficient $C_{i}$ is given by the ratio of existing links $E_{i}$ between its $k_{i}^{\text {out }}$ recurrences to a maximum possible number of such links, $\frac{1}{2} k_{i}^{\text {out }}\left(k_{i}^{\text {out }}-1\right)$. The clustering coefficient $C$ of the network is defined as the average over all vertices $i$ with out-degree larger than one

$$
C=\left\langle C_{i}\right\rangle_{i}=\left\langle\frac{2 E_{i}}{k_{i}^{\text {out }}\left(k_{i}^{\text {out }}-1\right)}\right\rangle_{i} .
$$

The values of the clustering coefficient for the different OFC catalogs are given in table [I. The original catalogs generate networks which are significantly more clustered than those generated by the shuffled catalogs. This effect decreases slightly for larger values of $\alpha$. A substantial amount of clustering seems to be related to the quasi-periodic attractors of the dynamics generating vertices with closed cascades of recurrences. This follows directly from the observation that the clustering is much less pronounced for those vertices with open cascades of recurrences (Table II). However, the latter events still retain a higher clustering than the networks for the shuffled OFC catalogs.

While a direct quantitative comparison with seismicity is not possible due to different catalog sizes and different mean degrees, the qualitative features are the same. In both cases, the catalogs show a much higher clustering compared to their respective shuffled counterpart.

\section{B. Temporal distances of recurrences}

The probability density function (PDF) $p^{s_{\text {th }}}(t)$ for the time intervals or waiting times $t$ associated with the recurrences or the edges of the network is another important characteristic. Fig. 8 shows this PDF for different OFC catalogs with $\alpha=0.2$. Despite some variation with $s_{\text {th }}$, all PDFs decay approximately as $1 / t$ up to the largest possible waiting time, determined by the finite time span of the catalog, with a superposed peak at a characteristic time $t_{c}$. As the inset of Fig. 8 shows, this characteristic time separates two slightly different regimes. While the PDFs decay almost exactly as $1 / t$ for $t>t_{c}$, the decay is rather $1 / t^{0.9}$ for $t<t_{c}$. Note also that $t_{c}$ does not depend on $s_{\text {th }}$. Such a characteristic time is expected since the activity shows quasi-periodic behavior as discussed in Section IV A 1. Moreover, $t_{c}$ depends on the dissipation parameter $\alpha$, as Fig. 9 shows. More specifically (as the inset of Fig. 9 shows), the characteristic time seems to be exactly $1-4 \alpha$, which is the natural period of the system: For periodic boundary conditions and relatively small $\alpha$, the system exhibits only trivial, perfectly synchronized topplings of size one and the activity returns to each site with period $1-4 \alpha$ [38]. It is even possible to identify secondary characteristic recurrence times in the inset of Fig. 9. corresponding to integer multiples of $1-4 \alpha$, again emphasizing the quasiperiodic behavior [45].

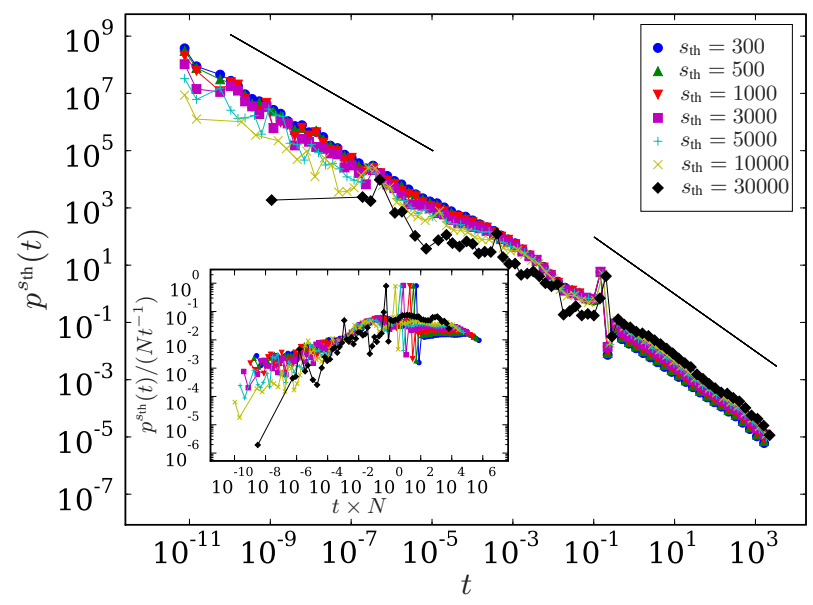

FIG. 8: (Color online) Distribution of the waiting times between events and their recurrences for different threshold sizes $s_{\text {th }}$, for $\alpha=0.2$. The solid line on the left is a power law with exponent -0.9 and on the right one with exponent -1 . Inset: Rescaled distributions with $N$ taken from table@

For the shuffled OFC catalogs, $p^{s_{\text {th }}}(t)$ is shown in Fig. 10 . As expected, the peak at the characteristic time scale $t_{c}$ is barely noticeable since the quasi-periodic behavior is basically absent in the shuffled catalog. Yet, all other qualitative features are preserved. In particular, the PDF is very different from the behavior of $p^{s_{\mathrm{th}}}(t)$ in the random CST case where a flat regime for small $t$ and $1 / t$ decay for large $t$ exist [18]. In the latter case, the transition time $t^{*}$ between the two regimes scales with the inverse of the rate of events $t^{*} \sim T / N$. We do not observe such a scaling for the shuffled OFC catalogs as follows from the inset of Fig. 10. While the data do collapse for large arguments, there is no good collapse for small arguments. This is due to the fact that there is a non-negligible probability that the time interval between subsequent events in the OFC model is arbitrarily small [36, 39] — in sharp contrast to the assumption of a Poisson process in the random CST case. 


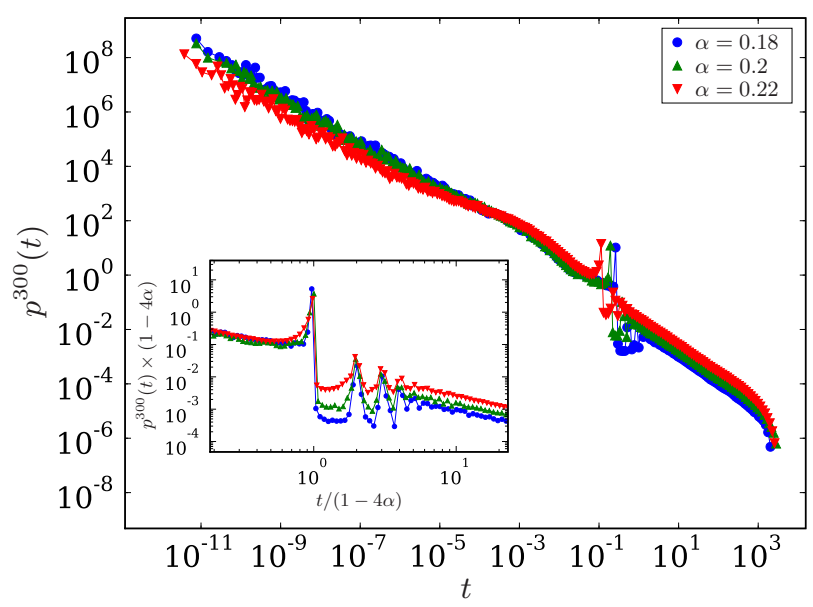

FIG. 9: (Color online) Distribution of the waiting times between events and their recurrences for $s_{\text {th }}=300$ and varying $\alpha$. Inset: Rescaled distribution, zoomed around the characteristic time region.

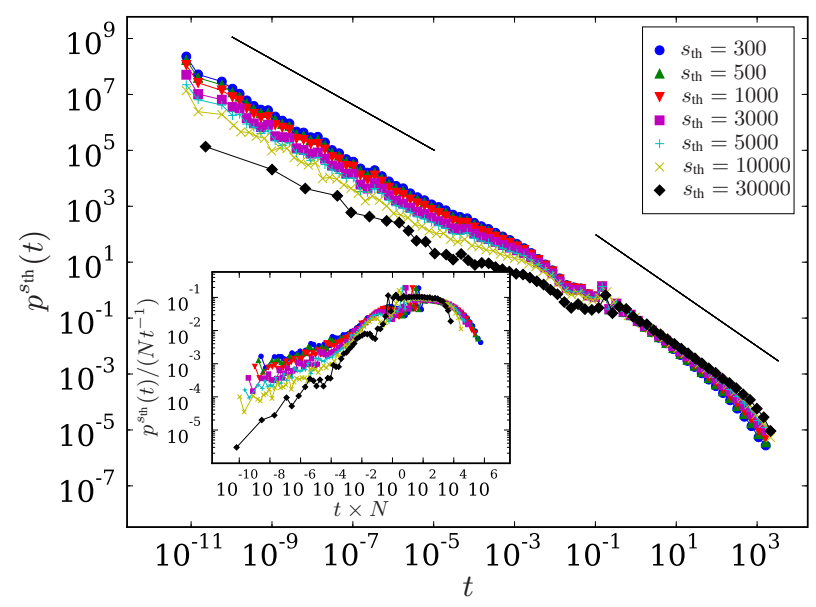

FIG. 10: (Color online) Distribution of waiting times between events and their recurrences for shuffled OFC catalogs with different threshold sizes $s_{\text {th }}$ and $\alpha=0.2$. The solid line on the left is a power law with exponent -0.9 and on the right one with exponent -1 . Inset: Rescaled distribution with $N$ taken from table

For seismicity $t^{*}$ - and indeed $p^{s_{\text {th }}}(t)$ - does not depend on $N$ (corresponding to a varying magnitude threshold) and there is also no equivalent to $t_{c}$ [18]. These observations clearly prove that the OFC model has certain features which are incompatible with seismicity and which strongly influence the generated spatiotemporal clustering of events.

\section{Temporal hierarchy of subsequent recurrences}

Another important aspect of the network of recurrences is the possible existence of hierarchies of recurrences. This can be captured by the ratios of the waiting times $t_{i} / t_{i+1}$ for subsequent recurrences belonging to the same originating event.
Here, it is assumed that the recurrences for a given event are ordered according to their time of occurrence such that the $i$-th recurrence is followed by the $i+1$-th recurrence for all ranks $i$. The corresponding PDFs are shown in Fig.111 For the OFC catalogs, panel (a) indicates that the PDFs vary with $i$. While they closely resemble a power law with exponent -0.85 in an intermediate regime for small $i$, deviations occur for larger $i$ 's. This is independent of $\alpha$ and $s_{\text {th }}$ (not shown). Independent of $i$, there is also a peak around $t_{i} / t_{i+1}=1$. As Fig. 11.(c) shows, there are only few discernible differences between the original and the shuffled catalogs: The peak is slightly more pronounced in the original catalog implying that subsequent recurrences are more likely to be separated by a very short time interval only, and the occurrence of smaller ratios for larger values of $i$ is more likely for the shuffled catalogs. A third and more pronounced but expected difference is that the kink related to $t_{C}$ around $t_{i} / t_{i+1} \sim 10^{-10}$ in the original catalog is absent in the shuffled catalog.

Even these small differences in the PDFs disappear if only open cascade of recurrences are considered, as shown in Fig. 11 (b) and (d). In all cases the PDFs depend very weakly on the value of $\alpha$, with all mentioned properties equally present in the range of $\alpha$ 's studied.
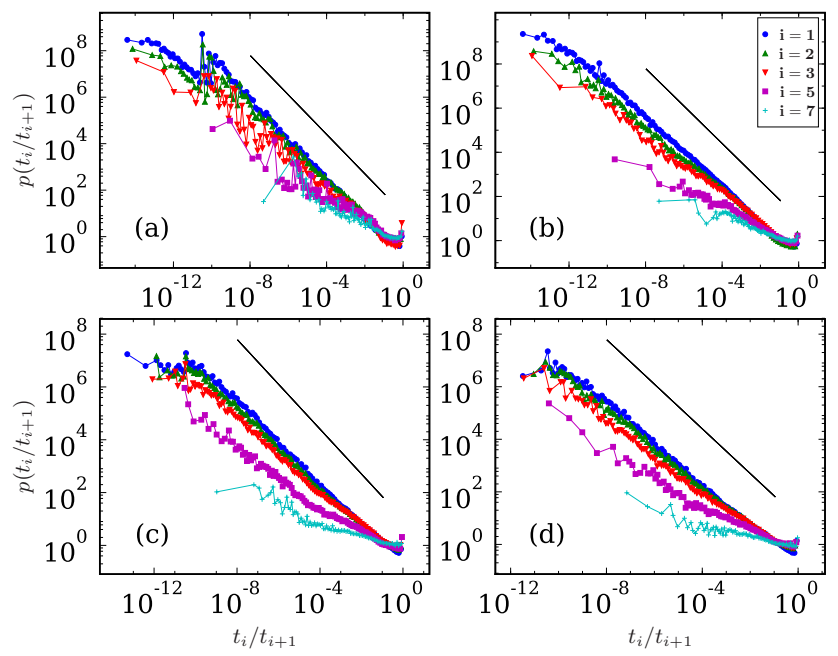

FIG. 11: Distribution of the waiting time ratios for subsequent recurrences, for $\alpha=0.2$ and $s_{\text {th }}=300$, for (a) the regular catalog, (b) only the "open" recurrences, (c) the shuffled catalog and (d) only the "open" recurrences for the shuffled catalog. In all plots the solid line is a power-law with inclination -0.85 .

For seismicity, the ratios $t_{i} / t_{i+1}$ for subsequent recurrences revealed a different non-trivial characteristic [18]. Their distribution showed two power-law regimes - in contrast to a single power law for the shuffled catalog. The spatiotemporal correlations responsible for these results do not seem to be present in the OFC model, as the above analysis shows. 


\section{Spatial distances of recurrences}

Exactly like the waiting times of recurrences, the spatial distances between an event and its recurrences can be obtained for the OFC model for further characterization and comparison with seismicity. The PDF of these distances $p^{s_{\text {th }}}(l)$ is shown in Fig. 12 for $\alpha=0.2$ and different size thresholds. The results for other values of $\alpha$ are qualitatively similar. The overall shape of the distributions is rather broad with cut-offs at short and long distances due to the discrete and finite lattice, respectively, and varies with $s_{\text {th }}$. Yet, for arguments less than the long distance cut-off two different regimes seem to be generally present. The inset of Fig. 12 shows an attempted scaling collapse according to the ansatz

$$
p^{s_{\mathrm{th}}}(l)=\frac{\left(l / s_{\mathrm{th}}^{\delta}\right)^{-\beta} F\left(l / s_{\mathrm{th}}^{\delta}\right)}{s_{\mathrm{th}}^{\delta}},
$$

with $\delta=0.3$ and $\beta=1.5$. If successful for arguments significantly less than the respective finite size cut-off, it would imply the existence of a characteristic distance dependent on $s_{\text {th }}$ with $l^{*} \sim s_{\text {th }}^{0.3}$ - qualitatively similar to seismicity and the random CST case as discussed below. However, as the inset of Fig.12 clearly shows, the data do not collapse as intended and a collapse does not seem to be possible at all. When only open cascades of recurrences are considered, as shown in Fig. 13, the PDFs are slightly different but there is no convincing collapse either. It does, however, show again that the statistics of those events that have closed cascades and predominantly belong to quasi-periodic attractors, are significantly different from the "open" ones.

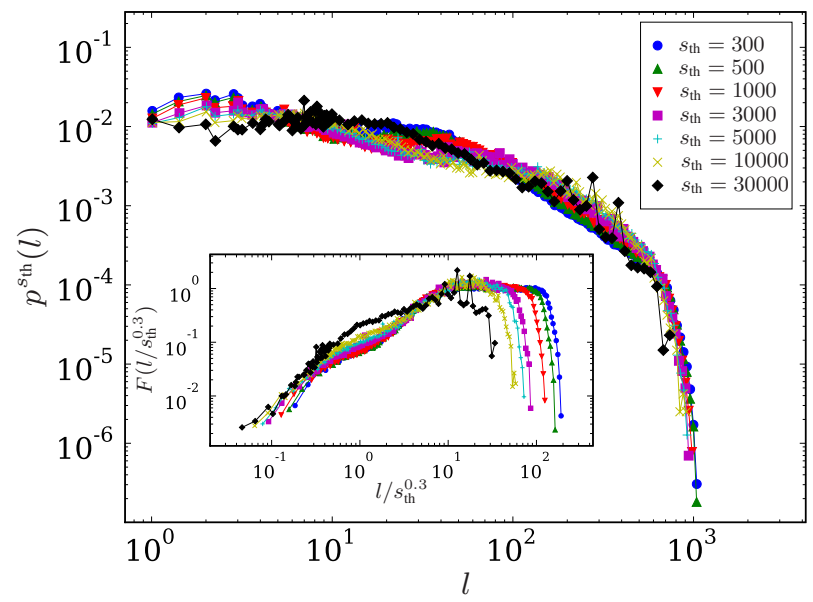

FIG. 12: (Color online) Distribution of recurrence distances for $\alpha=$ 0.2 and different threshold values $s_{\text {th. }}$. Inset: Distributions rescaled according to Eq. 22 with $\delta=0.3$ and $\beta=1.5$. Recurrences of distance $l=0$ are not shown, and were not used in the normalization.

For the shuffled OFC catalog the corresponding results for $p^{s_{\text {th }}}(l)$ are shown in Fig. 14, In this case, a collapse is indeed possible. Here, the collapse was done using the number of events $N$ of the respective catalog given in Table [. Due

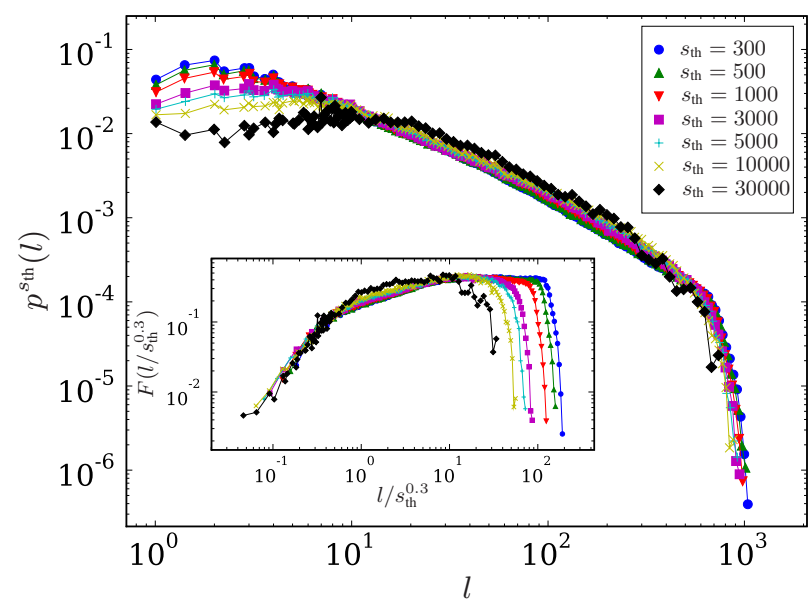

FIG. 13: (Color online) Distribution of recurrence distances for $\alpha=$ 0.2 and different threshold values $s_{\text {th }}$, but only considering "open" cascades of recurrences. Inset: Distributions rescaled according to Eq. (2) with $\delta=0.3$ and $\beta=1.35$.

to the absence of non-trivial correlations in the shuffled cata$\operatorname{logs}$, this is equivalent to the scaling with $s_{\text {th }}$ since $N \propto s_{\mathrm{th}}^{-0.8}$ as follows from the distribution of event sizes $P(s) \sim s^{-1.8}$. These findings can be directly compared to the random CST case for which $l^{*} \propto N^{-\gamma}$, where $\gamma=1 / D$ and $D$ is the underlying spatial dimension [18]. However, for the shuffled OFC catalog we find $l^{*} \propto N^{-0.18}$ which would imply $D \approx 5$. This is in sharp contrast to the fact that the OFC model is defined on a 2D lattice. This disagreement can be attributed to the assumption of continuous space made to derive the results in Ref. [18]. On a discrete lattice, when the characteristic distance approaches the fundamental lattice distance, the identification of the exponent with $1 / D$ cannot be expected. Despite this mismatch, the random CST process correctly predicts the power-law decay with exponent 1 for large distances of the shuffled OFC catalogs. Note that this decay clearly distinguishes the shuffled OFC catalogs from the original OFC catalogs.

The equivalence of the scaling of $p^{s_{\text {th }}}(l)$ with $s_{\text {th }}$ and with $N$ for the shuffled OFC catalogs discussed above is confirmed by Fig. 15 (c). There, the variation of $p^{s_{\text {th }}}(l)$ with the time length $\Delta t$ of the catalog is explicitly shown for $s_{\text {th }}=300$. Note that the $\mathrm{x}$ and $\mathrm{y}$ axis are rescaled with $\Delta t^{\gamma}$, where $\Delta t$ is the time length indicated in the legend. Not only the shuffled catalog varies with time (and hence $N$ ) for small distances but also the original catalog - even if only open cascades of recurrences are considered (see Fig. 15 (a) and (b)). In the latter case, the different curves can be collapsed using the same ansatz as for the shuffled catalog with $\gamma=0.37$, while the time dependence of the unmodified catalog seems to be more elaborate (it is shown unscaled in Fig. 15). Moreover, comparing Figs. 15 (b) and the inset of 13 allows to test the scaling relation $N^{\gamma} \propto s_{\mathrm{th}}^{-0.8 \gamma}$ in the case of open cascades of recurrences. Indeed, we have $0.8 \times 0.37 \approx 0.3$ assuming $N \propto \Delta t$. This would imply that the dependence of $p^{s_{\text {th }}}(l)$ 


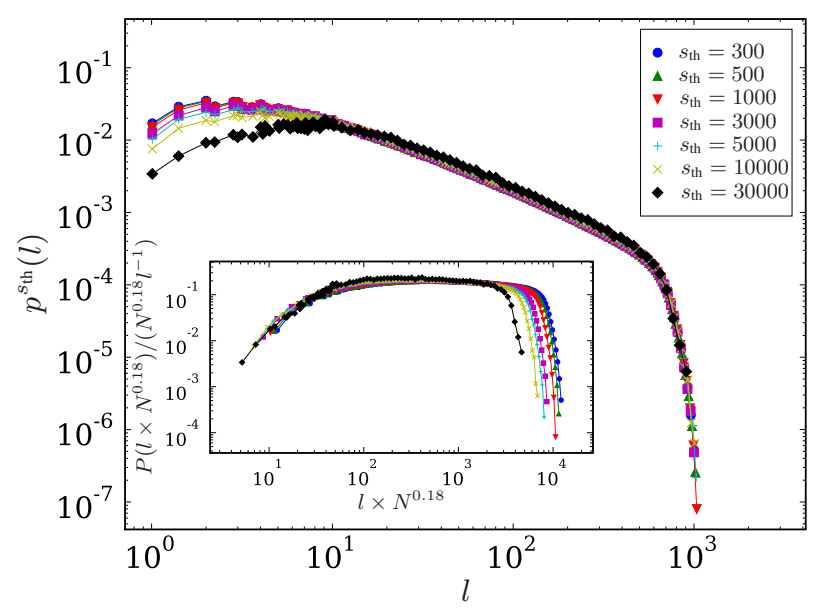

FIG. 14: (Color online) Distribution of recurrence distances for the shuffled OFC catalog with $\alpha=0.2$ and different threshold values $s_{\text {th }}$. Inset: Distributions rescaled according to the number of events (see Table $\square$ ) as indicated in the legend.

on $s_{\text {th }}$ is simply due to the variation in $N$. Yet, the fact that $p^{s_{\text {th }}}(l)$ does not obey scaling suggests that the situation is more complicated and that there is a non-trivial dependence on $s_{\mathrm{th}}$.

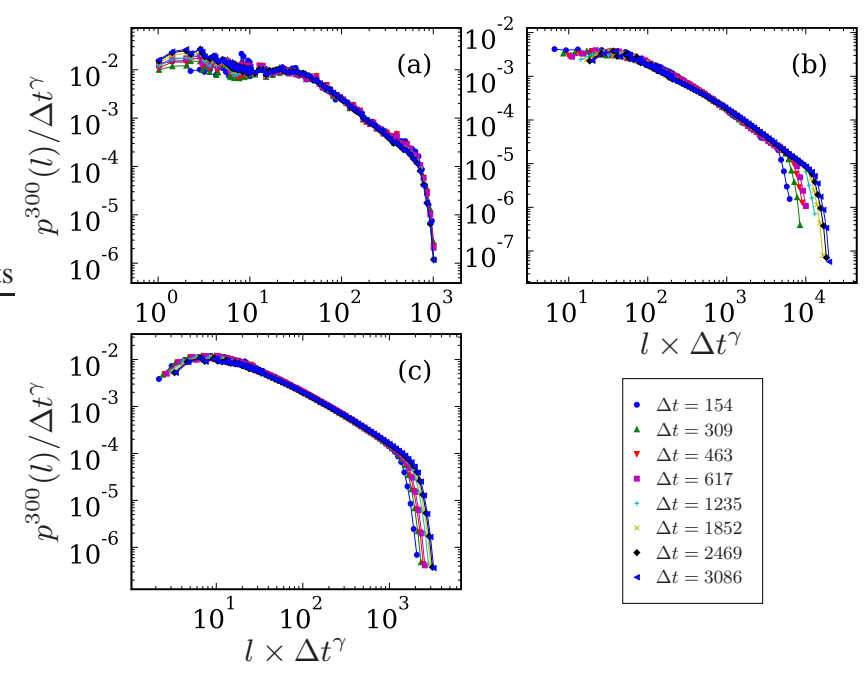

FIG. 15: (Color online) Distribution of recurrence distances for $\alpha=$ 0.2 and $s_{\text {th }}=300$, for an increasingly larger potion of the catalog $\Delta t$, re-scaled as indicated in the legend, for (a) the regular catalog ( $\gamma=0$, i.e. no scaling), (b) only the "open" recurrences $(\gamma=0.37)$ and (c) the shuffled catalog $(\gamma=0.18)$.

For seismicity, $p^{\text {st }}(l)$ shows a characteristic distance $l^{*}$, after which the distribution decays as a power-law with an exponent close to one [18]. The value of $l^{*}$ depends only on the lower magnitude threshold and, in particular, does not change with time (or $N$ ) for fixed $m$. More specifically the scaling of this characteristic distance obeys the ansatz $l^{*}(m) \propto 10^{0.45 m}$, where $m$ is the lower threshold magnitude. This allows one to identify $l^{*}$ with the rupture length of an earthquake - its spatial extent - even though it is explicitly neglected in the point process description. In particular, it directly relates the underlying microscopic dynamics to the statistical properties of the network of recurrences - one of the main achievements for seismicity. As shown above, the OFC model does not successfully reproduce these features. While the characteristic distance does depend on the lower threshold size, no simple scaling ansatz can be identified (Eq.(2)), even when only "open" cascades of recurrences are considered. More importantly, the characteristic distance decreases with time in both cases.

\section{Spatial hierarchy of subsequent recurrences}

The spatial hierarchy of recurrences can be captured by the distribution of distance ratios $l_{i+1} / l_{i}$ for subsequent recurrences belonging to the same originating event. Here, it is assumed that the recurrences for a given event are ordered according to their time of occurrence such that the $i$-th recurrence is followed by the $i+1$-th recurrence. The corresponding PDFs are shown in Fig. 16 for $\alpha=0.2$ and $s_{\text {th }}=300$ but the PDFs seem to be independent of the specific values of $\alpha$ and $s_{\text {th }}$ in the range we studied. We have also included the case $i=0$ for which we use $l_{0}=\sqrt{2} L_{b u l k}$. Note that $l_{1}$ corresponds to the distances between subsequent events and that in this case there is a cut-off at large arguments due to the finite lattice size. For all $i>0$ the PDFs shown in Fig. 16 seem to be independent of $i$. For the original OFC catalog (Fig.16(a)), the PDFs increase for small arguments and then become flat for larger ones, before increasing again as the ratio approaches 1 . The same behavior is observed when only open cascades of recurrences are considered (Fig. 16(b)).

The situation is different for the shuffled OFC catalogs. As shown in Fig.16(c), all PDFs increase as a power-law with exponent 1 . This is similar to the random CST model which predicts $p(x)=D x^{D-1}$, independent of $i$, with $x=l_{i+1} / l_{i}[18]$. For the shuffled OFC catalogs, this would imply a spatial dimension $D=2-$ exactly as expected. The same behavior is observed when only open cascades of recurrences are considered (Fig. 16(d)). The comparison of the OFC catalogs and their shuffled counterparts suggests that the distribution of ratios between subsequent recurrence distances indeed captures non-trivial aspects of the spatiotemporal dynamics.

All cases are however very different from seismicity, where the ratios all decay as a power-law with exponent $\sim 0.6$.

\section{DISCUSSION \& CONCLUSION}

We have studied the network of recurrences of the OFC model, with the objective of characterizing its causal structure and comparing it to seismicity. We have found that, in agreement with previous results [23, 24, 25], the model does exhibit some non-trivial features observed in seismicity, but it comes short of a complete qualitative description of several robust features of spatiotemporal clustering captured by the 

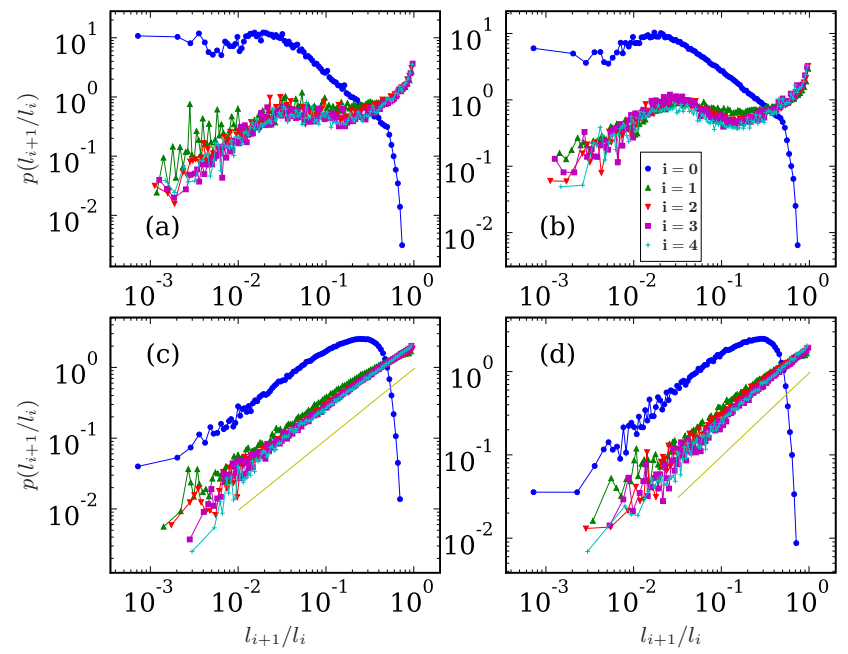

FIG. 16: Distribution of ratios between subsequent recurrence distances for $\alpha=0.2$ and $s_{\text {th }}=300$, for (a) the regular catalog, (b) only the "open" recurrences, (c) the shuffled catalog and (d) only the "open" recurrences for the shuffled catalog. The solid line is a power law with exponent 1.

used method. More specifically, the model reproduces central topological features of the recurrences network, which are the degree distributions and high clustering, as well as the absence of non-trivial degree correlations and some other partial aspects of the recurrence distance and time distributions. However, the two most important properties of the dynamics of seismicity identified by this recurrence approach are, firstly, that the time (distance) distributions are independent of magnitude thresholds (time), and secondly, that the distance statistics provides an independent assessment of the rupture length of earthquakes. The OFC model fails to reproduce the first feature, as both the time and distance distributions vary with magnitude threshold and time, respectively. The second feature is also not reproduced. While there is a dependence of the distance distribution on the size of the events, there is no scaling which would allow to identify a rupture length. One third, and more subtle, characteristic of the recurrences which is not reproduced by the OFC model is the hierarchy of recurrences, given by the distribution of ratios of time and distance between consecutive recurrences, which in the case of seismicity deviates significantly from the random case. Moreover, the whole analysis exposes another crucial aspect of the OFC model, which is the elevated occurrence of epicenters at distance zero from one another, tightly connected to quasiperiodicity and well distinct from seismicity.

Obviously, the OFC model was not intended to be a complete description of seismicity. It was mainly proposed as a conceptual model and a possible origin of the GutenbergRichter law from a SOC point of view. Also, like the Burridge-Knopoff spring-block model, it aims to model the dynamics of a single fault, and not the complex interplay of different fault structures as observed in reality. Despite these limitations, the OFC model is capable of showing a rich dynamics, reproducing even some more subtle features of the spatiotemporal clustering of earthquakes.

\section{ACKNOWLEDGMENTS}

We thank the organizers of the Workshop on "Dynamics on Complex Networks and Application" at the Max-PlanckInstitute for Physics of Complex Systems, Dresden, Germany, where this work was initiated. We also thank Peter Grassberger for his comments on the manuscript. Part of this work was supported by Fundação de Amparo à Pesquisa do Estado de São Paulo (FAPESP), process number 03/03429-6.
[1] D. L. Turcotte, Fractals and chaos in geology and geophysics (Cambridge University Press, Cambridge, UK, 1997), 2nd ed.

[2] S. Stein and M. Wysession, An introduction to seismology, earthquakes, and earth structure (Blackwell Publishing, Oxford, UK, 2002).

[3] F. Mulargia and R. J. Geller, eds., Earthquake science and seismic risk reduction, NATO Science Series (Kluwer Academic Publishers, Dordrecht, NL, 2003).

[4] J. B. Rundle, D. L. Turcotte, R. Shcherbakov, W. Klein, and C. Sammis, Review of Geophysics 41, 1019 (2003).

[5] P. Bak, K. Christensen, L. Danon, and T. Scanlon, Physical Review Letters 88, 178501 (2002).

[6] A. Helmstetter, G. Ouillon, and D. Sornette, Journal of Geophysical Research 108, 2483 (2003).

[7] A. Corral, Physical Review Letters 92, 108501 (2004).

[8] J. Davidsen and C. Goltz, Geophysical Research Letters 31, L21612 (2004).

[9] M. Baiesi and M. Paczuski, Physical Review E 69, 066106 (2004).

[10] R. Shcherbakov, D. L. Turcotte, and J. B. Rundle, Geophysical
Research Letters 31, L11613 (2004).

[11] J. Davidsen and M. Paczuski, Physical Review Letters 94, 048501 (2005).

[12] A. Helmstetter, Y. Y. Kagan, and D. D. Jackson, Journal of Geophysical Research 110 (2005).

[13] V. N. Livina, S. Havlin, and A. Bunde, Physical Review Letters 95, 208501 (2005).

[14] G. Molchan and T. Kronrod, Geophysical Journal International 162, 899 (2005).

[15] R. Shcherbakov, D. L. Turcotte, and J. B. Rundle, Bulletin of the Seismological Society of America 96, 376 (2006).

[16] K. R. Felzer and E. E. Brodsky, Nature (London) 441, 735 (2006).

[17] J. Davidsen, P. Grassberger, and M. Paczuski, Geophysical Research Letters 33, L11304 (2006).

[18] J. Davidsen, P. Grassberger, and M. Paczuski, Physical Review E p. submitted (2006).

[19] R. Albert and A.-L. Barabasi, Review of Modern Physics 74, 47 (2002).

[20] M. E. J. Newman, SIAM Review 45, 167 (2003). 
[21] Z. Olami, H. J. S. Feder, and K. Christensen, Physical Review Letters 68, 1244 (1992).

[22] K. Christensen and Z. Olami, Journal of Geophysical Research 97, 8729 (1992).

[23] S. Hergarten and H. J. Neugebauer, Physical Review Letters 88 , 238501 (2002).

[24] A. Helmstetter, S. Hergarten, and D. Sornette, Physical Review E 70, 046120 (2004).

[25] T. P. Peixoto and C. P. C. Prado, Physical Review E 74 (2006).

[26] P. Bak, How nature works (Copernicus, New York, 1996).

[27] H. J. Jensen, Self-organized criticality (Cambridge University Press, Cambridge, UK, 1998).

[28] P. Bak, C. Tang, and K. Wiesenfeld, Physical Review Letters 59, 381 (1987).

[29] J. X. Carvalho and C. P. C. Prado, Physical Review Letters 84, 4006 (2000).

[30] S. Lise and M. Paczuski, Physical Review E 63, 036111 (2001).

[31] S. Lise and M. Paczuski, Physical Review E 64 (2001).

[32] G. Miller and C. J. Boulter, Physical Review E 66 (2002).

[33] C. J. Boulter and G. Miller, Physical Review E 68, 056108 (2003).

[34] F. Wissel and B. Drossel, Physical Review E 74, 066109 (2006).

[35] R. Burridge and L. Knopoff, Bulletin of the Seismological Society of America 57, 341 (1967).

[36] J. Davidsen and H. G. Schuster, Physical Review E 62, 6111 (2000).

[37] A. A. Middleton and C. Tang, Physical Review Letters 74, 742 (1995).

[38] P. Grassberger, Physical Review E 49, 2436 (1994).

[39] B. Drossel, Physical Review Letters 89, 238701 (2002).

[40] The area $a$ of an avalanche can in principle be different from its size $s$, since sites can topple more than once. However, there is a transition value of $\alpha$, below which multiple topplings are impossible. This value can be obtained by considering the extremal situation where a site with tension 1 is about to topple, whose neighbors also have tension 1 . After the site topples, it will also cause the topplings of the neighbors, which will then return tension to original site. The amount of tension returned is $4 \alpha(1+\alpha)$. If this value is below 1 , then the original site will not topple a second time, which means that no site in any other situation will topple a second time. Thus, the transition value is $\alpha=(\sqrt{2}-1) / 2 \approx 0.207$. Below this value, $a=s$ for any event. Simulations show that even when the value of $\alpha$ is slightly above this transition, the occurrence of multiple topplings is negligible. For the range of $\alpha \in[0.22,0.18]$ studied here, multiple topplings play no detectable role.

[41] The sizes of the discarded transients were $\sim 10^{10}, \sim 7 \times 10^{9}$ and $\sim 4 \times 10^{9}$ iterations for $\alpha=0.18,0.2$ and 0.22 , respectively.

[42] A network is composed of a set of discrete elements called vertices (or nodes) and a set of pairs of vertices (which can be ordered or not), called edges (or links), which may describe some relationship between two vertices. Two vertices are neighbors if there is a edge between them. The number of neighbors of a given vertex is called the degree of the vertex. If the edges of the network are directed, then the vertex can have in-neighbours and out-neighbours, depending on the direction of the corresponding edge, and therefore also an in-degree and an out-degree. See [20] for an extensive and detailed review of these concepts.

[43] The ratio of open vs. closed cascades of recurrences, after some initial fluctuations, remains stable as the catalog grows, at least for the lengths of time studied. However, since the size of the system is finite, eventually the number of closed cascades should overcome the number of open ones. The observed stability shows that the obtained catalogs are still not affected by this finite size effect.

[44] The distributions are even independent of $\alpha$ or $s_{\text {th }}$.

[45] The shape of $p^{s_{\text {th }}}(t)$ does not change if recurrences belonging to closed cascades are excluded. In particular, the characteristic time scale $t_{c}$ is still present though slightly less pronounced. 